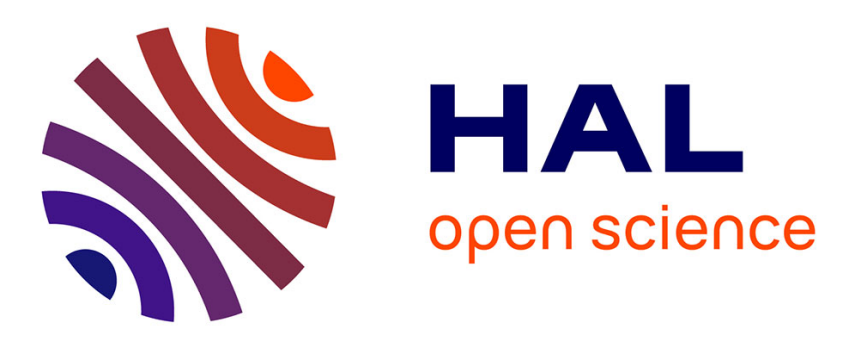

\title{
Des distributeurs toujours plus proches du consommateur? Le cas du drive alimentaire
}

Dany Vyt, Magali Jara, Olivier Mevel, Thierry Morvan, Nélida Morvan

\section{To cite this version:}

Dany Vyt, Magali Jara, Olivier Mevel, Thierry Morvan, Nélida Morvan. Des distributeurs toujours plus proches du consommateur? Le cas du drive alimentaire. Revue management \& avenir, 2017, 93 (3), pp.141-160. 10.3917/mav.093.0141 . halshs-01806406

\section{HAL Id: halshs-01806406 https://shs.hal.science/halshs-01806406}

Submitted on 3 Sep 2018

HAL is a multi-disciplinary open access archive for the deposit and dissemination of scientific research documents, whether they are published or not. The documents may come from teaching and research institutions in France or abroad, or from public or private research centers.
L'archive ouverte pluridisciplinaire HAL, est destinée au dépôt et à la diffusion de documents scientifiques de niveau recherche, publiés ou non, émanant des établissements d'enseignement et de recherche français ou étrangers, des laboratoires publics ou privés. 


\title{
Des distributeurs toujours plus proches du consommateur? Le cas du drive alimentaire
}

\author{
Dany Vyt \\ Maître de Conférences, Centre de Recherche en Economie et Management (CREM UMR \\ CNRS 6211), IGR-IAE Rennes, dany.vyt@univ-rennes1.fr
}

\section{Magali Jara}

Maître de Conférences, Laboratoire d'Economie et de Management (LEMNA), IUT de Saint Nazaire-Université de Nantes, magali.jara@ univ-nantes.fr

\section{Olivier Mevel}

Maitre de conférences, Université de Brest/ICI - IUT de Brest, Olivier.mevel@univ-brest.fr

\section{Thierry Morvan}

Maitre de conférences, Université de Rennes 1/ICI - IUT de Saint Malo

Thierry.morvan@univ-rennes1.fr

\section{Nélida Morvan}

Maitre de conférences, Université de Rennes 1/I IUT de Saint Malo nelida.morvan@univ-rennes1.fr 


\title{
Des distributeurs toujours plus proches du consommateur? Le cas du drive alimentaire
}

\section{Résumé}

Cette recherche quantitative s'intéresse aux acheteurs de drive alimentaire et spécifiquement à leur fidélité à l'enseigne. Leurs comportements de fidélité seront analysés par le prisme de la proximité d'accès, fonctionnelle et relationnelle. Les résultats confirment que le drive a bien pour vocation de consolider la relation de service établie par l'enseigne avec le client. Ce dernier perçoit le drive comme un service complémentaire et a tendance à rester plutôt fidèle à son enseigne.

Mots-clés : Fidélité, Drive, Grande distribution Multicanal, Proximité.

\begin{abstract}
This quantitative research focuses on the food "click and collect" buyers and their loyalty towards their retailer. User loyalty will be analyzed through the prism of the access, functional and relational proximity. The results confirm that the "click and collect" has intended to consolidate the service relationship established by the retailer with the customer. He actually perceives the "click and collect" as an additional service and is loyal to his retailer.
\end{abstract}

Key-words: Click and collect, loyalty, proximity, multichannel, retailing.

\section{Introduction}

Depuis 2001, les consommateurs français se voient proposer une nouvelle manière de réaliser leurs achats: le drive (ou «click and collect»). Les réseaux exploitent les synergies du $e$ shopping et du drive-in système. Le principe du drive est simple : le shopper commande en ligne depuis un site internet dédié ou une application pour téléphone mobile et vient lui-même chercher ses achats. Pour Rouquet (2014), il s'agit de «tout format de distribution qui permet un transfert direct des produits achetés par le client de leur lieu de stockage jusqu'à son véhicule ». Le shopper devient alors un «client transporteur»(Marousseau, 2013). Le consommateur bénéficie de la combinaison des atouts du magasin principal avec ceux d'un magasin en ligne (Beck et Rygl, 2015). L'introduction de ce nouveau canal combinée à la multiplication des points de contact entre le distributeur et son client bouleverse les habitudes d'achat des shoppers. En 2016, le drive affiche une part de marché en valeur de 5,5\% ${ }^{1}$ et pèse désormais plus de 4,5 milliards d'euros sur les seuls produits de grande consommation et le frais en libre-service ${ }^{2}$ (PGC-FLS). L'objectif de cet article est d'étudier l'impact de ce

\footnotetext{
${ }^{1}$ Source: Kantar World Panel/ Distri Book, Février 2017.

${ }^{2}$ Source : Nielsen Scan Track, Homesacon, Trade dimensions, juillet 2016.
} 
nouveau canal sur la fidélité à l'enseigne. En effet, $16 \%$ des foyers français viennent au drive chaque trimestre et $6 \%$ utilisent le drive comme magasin principal ${ }^{3}$. A l'heure où les premiers drives ferment, de nombreuses questions demeurent. Renforce-t-il l'attachement à l'enseigne par une expérience de cross shopping valorisante ou bien nous fait-il changer d'enseigne, faisant de nous ce que d'aucuns nomment des passagers clandestins (Van Ball et Dach, 2005)?

Ce papier se structure de la manière suivante. La première partie situe le drive dans le commerce ubiquitaire puis propose un cadre théorique de référence en vue de comprendre la formation des perceptions des clients du drive, préalables à leurs comportements de fidélité. Une deuxième partie détaille l'analyse quantitative mise en place pour évaluer la fidélité des consommateurs à l'enseigne dans le contexte particulier du drive. Les comportements hétérogènes des utilisateurs de drive sont analysés par le prisme de la proximité d'accès, fonctionnelle et relationnelle (Bergadaà et Del Bucchia, 2009). Cette lecture des résultats met à jour plusieurs profils de clients fidèles conduisant à les segmenter en trois groupes homogènes grâce à une analyse typologique (classification ascendante hiérarchique). Les résultats obtenus sont ensuite discutés et impliqueront des recommandations aux professionnels de la distribution.

\section{Le drive dans le développement du commerce ubiquitaire}

\subsection{Le drive dans la stratégie cross canal des réseaux}

Alors que le multicanal se matérialise par une juxtaposition des canaux, sans véritable synergie, le cross canal symbolise la multiplication des points d'accès aux produits et donc une coordination et une complémentarité des canaux entre eux (Picot Coupey, Huré et Piveteau, 2016). Le cross canal replace le consommateur au cœur de la démarche des réseaux de distribution et se veut d'une grande utilité dans la création de valeur pour le shopper (Payne et Frow, 2004). Le site internet devient un incontournable dans la gestion de la relation client (Seck, Fulconis et Paché, 2014). Avec l'introduction d'internet dans le processus d'achat des clients, les distributeurs évoluent d'une stratégie unique brick and mortar, fondée sur un réseau physique de points de vente, vers un système click and mortar and combinant la force de chacun des canaux de distribution, et générant ainsi des ventes additionnelles (Dinner, Van Heerde et Neslin, 2014). Ainsi, le consommateur peut-il combiner de multiples formes d'achat. En plus d'effectuer ses achats en point de vente traditionnel, le shopper peut aujourd'hui opter pour un achat en ligne avec livraison à domicile, ou un retrait en magasin.

Trois modèles dominants de service au volant coexistent : 1) le drive déporté ou isolé (drive pure player), 2) le drive accolé ainsi que 3) le service drive. Dans le cas du drive déporté, il n'existe aucun magasin physique, mais uniquement des entrepôts, faisant office de points de retrait. L'enseigne Chronodrive (Groupe Auchan) s'inscrit dans cette logique de pure player, puisque l'intégralité de son réseau se fait sous forme de points de retrait. Concernant le drive accolé, cela revient à implanter à proximité d'un point de vente existant un entrepôt de stockage et de préparation de commandes. La préparation des commandes s'opèrent à partir des articles présents en magasin: on parle alors de picking. Plusieurs enseignes d'hypermarchés privilégient cette forme de drives. Ainsi l'intégralité du parc d'hypermarchés Cora développe des drives accolés. Enfin, dans le troisième cas de figure, l'idée consiste à proposer un service drive à partir d'un magasin existant. Les points de vente développent ce

\footnotetext{
${ }^{3}$ Source : Nielsen Scan Track, Homescan, Trade dimensions, juillet 2016
} 
système de drive en créant ou en allouant une partie de leur surface au retrait des marchandises, équipée de bornes de retrait. Les grandes surfaces spécialisées telles que Decathlon ou encore Cache-Cache recourent à cette forme de drive. Si cela permet de répondre aux exigences actuelles du shopper en se libérant des achats corvée en magasin physique (Colla et Lapoule, 2015) et en maîtrisant la gestion de son temps d'achat aux consommateurs et de disposer d'un choix de produits plus large et plus profond, elle se veut coûteuse en main d'œuvre. Quelle que soit la complexité de la formule de service mise en œuvre, ce nouveau canal de distribution permet aux réseaux de lever le problème du dernier kilomètre (Colla et Lapoule, 2011; Picot et al, 2010; Punakivi et al., 2001) tout en permettant un redéploiement efficace de la couverture territoriale.

En dépit de sa récence, le drive séduit chaque année de plus en plus de consommateurs : ainsi, 26,2\% des ménages se sont laissés tentés par le drive en 2016. Désormais ce modèle de développement capte plus de 5,5\% de parts de marché, ${ }^{4}$ se posant ainsi comme une offre complémentaire des réseaux physiques.

\subsection{La complémentarité des canaux en question}

La coordination et l'intégration de l'ensemble des canaux représentent les enjeux majeurs du développement cross canal des réseaux (Seck, 2010 ; Colla et Lapoule, 2012, 2015 ; Picot Coupey, Huré et Piveteau, 2016). La fluidité du parcours d'achat cross canal devient le maître mot. Dans quelle mesure le drive représente-t-il un nouveau gisement de croissance interne pour les réseaux? A titre d'exemple, le développement d'un programme de fidélité cohérent entre le point de vente physique et le drive, peut améliorer la fidélité des shoppers à leur enseigne (Colla et Lapoule, 2015). Il s'agit alors de tirer profit des informations relatives aux clients pour optimiser l'offre de service (Seck, 2010). Dans la même veine, la combinaison d'une publicité traditionnelle avec une publicité en ligne peut générer des effets positifs sur l'ensemble de l'enseigne (Dinner, Van Heerde et Neslin, 2014). Concernant le drive, le picking des drives accolés ne doit pas désorganiser le point de vente physique dans sa gestion quotidienne (Seck, Fulconis et Paché, 2014). Pour ces auteurs, l'enjeu du cross canal réside dans une organisation transversale des différents canaux.

En dépit de nombreuses synergies positives entre les canaux de distribution, de nombreuses questions demeurent. Le drive alimentaire se pose-t-il en canal utilitaire, véritable catalyseur des ventes, ou bien représente-t-il un parfait substitut au point de vente physique ? La largeur ainsi que la profondeur des assortiments en ligne, notamment des drives solos, ne rivalisent nullement avec la taille des assortiments en magasin. L'étendue de choix dans les magasins étant nettement plus importante que sur le site des drives (leurs références représentent en moyenne entre 20 à $25 \%$ de l'assortiment d'un hypermarché), l'absence de congruence dans le choix proposé au shopper ne peut-il pas induire, voire amplifier, le butinage vers les concurrents (Bezes, 2012) ? Véritable témoin de la diffusion des innovations technologiques, la multiplication des points d'accès aux produits pose de nouveaux enjeux pour les réseaux de distribution. Dès 2007, Rosenbloom pointe les limites d'une stratégie multicanale et rappelle alors que la démultiplication des canaux n'implique pas nécessairement un accroissement du nombre de clients. Si la taille du marché potentiel stagne, l'ajout d'un nouveau canal entraîne des coûts supplémentaires pour l'enseigne et donc une baisse de la rentabilité (Van Baal et Dach, 2005). Pire, d'aucuns dénoncent une cannibalisation possible entre les canaux (Rosenbloom, 2007 ; Seck, 2010 ; Colla et Lapoule, 2012, 2015). Le développement de l'un se faisait alors au détriment de l'autre. Alors que les

\footnotetext{
${ }^{4}$ Source: Distribook, Février 2017.
} 
coûts de changement sont relativement faibles pour le shopper, chaque drive étant à un clic de son concurrent, ce canal de distribution modifie-t-il la fidélité des consommateurs à l'enseigne ? Ce consommateur mixte, qui utilise l'ensemble des canaux de distribution, doit-il faire l'objet d'une attention particulière comme le suggère Vanheems (2009) ? Si plusieurs recherches démontrent que le shopper cross canal se veut plus rentable que son homologue mono-canal (Rangaswamy 2005) qu'en est-il sa fidélité ? Wallace, Giese et Johnson (2004) démontrent que la complémentarité des canaux accroît la satisfaction du shopper le conduisant ainsi à être fidèle à l'enseigne. Heitz, Douard et Cliquet (2015) étudient le cas particulier des drives alimentaires et tempèrent cette relation. La fidélité à l'enseigne se vérifie pour les consommateurs qui perçoivent le drive comme un service annexe à l'hypermarché, avec une utilisation ponctuelle alors que la volatilité des consommateurs est mise à jour dans le cas d'un usage plus régulier du service drive. C'est ainsi qu'il convient de poursuivre ces contributions en étudiant cette relation de fidélité à l'enseigne des acheteurs cross canal. Choisissent-ils le drive de leur enseigne habituelle ou bien la fréquentation d'un drive est-elle contingente d'autres considérations ?

\section{La fidélité au drive et le rôle majeur de l'enseigne}

\subsection{Définition et mesures de la fidélité}

Plusieurs travaux proposent une grille d'analyse des différentes théories de la fidélité. A travers ce prisme, deux écoles émergent. Le premier, de tradition béhavioriste, définit la fidélité du client à partir de ses comportements d'achat, "Il n'y a pas lieu de considérer ce que le client pense, ni la manière dont fonctionne son système nerveux central. Son comportement est l'expression même de sa fidélité », (Tucker, 1964). Ce type de fidélité se mesure fréquemment à travers les achats répétés (Ehrenberg, 2000) ou d'intention de ré-achat (Chaudhuri et Holbrook, 2001 ; Sirdeshmukhet, Sing et Sabol, 2002). Toutefois, le choix de la mesure représente le point de divergence de ce courant de recherche. Le second s'inspire des approches cognitives pour lesquelles la fidélité est présente chez le client avant même de passer à l'acte (Jacoby et Kyner, 1973). L'introduction de la variable temporelle enrichit et précise la définition du concept. Dans le cadre de cette recherche, nous nous concentrons sur l'approche comportementale de la fidélité afin d'étudier le comportement réel des consommateurs (Meyer-Waarden, 2007).

$\mathrm{Ne}$ disposant d'aucun cadre théorique de référence sur lequel engager cette réflexion centrée sur le drive, nous proposons de rejoindre, dans un premier temps, les travaux de référence dédiés au comportement du consommateur envers un produit marqué. Les contributions de Tybout et Hauser (1981) sous-tendent en effet les approches comportementales de la fidélité. Les caractéristiques du produit traitées par le consommateur forment ses perceptions du produit marqué. Celles-ci sont modérées par ses caractéristiques individuelles et des variables externes. Si ce qu'il perçoit du produit marqué maximise son utilité, alors le consommateur nourrit des préférences envers ce dernier. Le choix final et répété envers le produit marqué résulte de ces préférences modérées par des considérations de prix et de facteurs environnementaux imprévisibles.

Il convient désormais d'approfondir cette approche comportementale par les contributions en distribution, particulièrement celles dédiés à la formation des images (de la marque, des enseignes ou encore des magasins) en vue de comprendre la formation des perceptions conduisant aux comportements de fidélité au drive.

\subsection{La fidélité au drive se construit à travers l'image de son enseigne}


Considérons le drive comme une extension nouvelle de son enseigne au même titre qu'un magasin ou qu'une marque de distribution. L'enseigne de distribution ayant une activité de service, cela implique que ses extensions reflètent cette particularité (Berry, 1986 ; Berry, 2000 ; Burt et Sparks, 2002). Le transfert d'image ou d'associations d'une manière générale entre l'enseigne et la formule drive peut s'apprécier par analogie au transfert entre l'enseigne et sa marque.

La marque peut en effet s'envisager comme un réseau sémantique formé de catégories stockées dans la mémoire des consommateurs (Mervis et Rosch, 1981), où chaque catégorie se forme de plusieurs objets distincts mais perçus comme équivalents (Cegarra et Merunka, 1993). Des transferts d'image entre les objets se produisent au sein d'une même catégorie cognitive ainsi que des préférences généralisées (Fry, 1967) et des attitudes positives à l'égard de l'ensemble des objets de la catégorie (Boush et Loken, 1991). Autrement dit, le consommateur se sert des associations de l'image de l'enseigne pour déduire ou inférer une partie des associations de l'image de la nouvelle formule commerciale. Il s'agit d'un effet de halo, décrit par Alba et Hutchinson (1987) entre les entités enseigne - points de vente marque de distributeur - drive dès lors qu'il existe une similarité ou une liaison logique perçue entre les quatre entités (par exemple un nom identique entre ces entités). C'est ainsi, que nous pouvons émettre l'idée que l'image du drive se construit en partie des associations d'image de son enseigne, des points de vente et de la marque du distributeur. De là, il semblerait cohérent sur la base de perceptions positives envers le drive et son enseigne, que le client adopte un comportement de ré-achat et de recommandation auprès du drive de son enseigne habituelle.

L'enjeu principal de ce travail consiste à présent à évaluer la fidélité du consommateur cross canal à son enseigne dans le contexte particulier du drive alimentaire.

\section{Etude empirique}

L'étude empirique a pour objectif de mesurer de manière descriptive, à ce stade, les comportements de fidélité envers le drive et son enseigne.

\subsection{Recueil de données}

L'enquête empirique se déroule auprès d'un territoire spécifique la Bretagne qui par le fait qu'il s'agit d'une région motrice dans le développement des drives en France et qui apparait comme l'une des régions les plus denses avec plus de 1 drive pour 13000 habitants (contre 1 drive pour 23514 habitants en moyenne) ${ }^{5}$. Nous avons soumis un questionnaire auprès d'un échantillon de convenance de 701 d'utilisateurs de drive répartis comme suit dans les quatre départements bretons (tableau 1).

Tableau 1 : Répartition de l'échantillon par département

\begin{tabular}{|c|c|c|c|}
\hline & Effectifs & $\begin{array}{c}\text { Pourcentage } \\
\text { échantillon }\end{array}$ & $\begin{array}{c}\text { Répartition des } \\
\text { habitants }\end{array}$ \\
\hline Côtes d'Armor & 200 & $28,5 \%$ & $18,39 \%$ \\
\hline Finistère & 218 & $31,1 \%$ & $27,84 \%$ \\
\hline Ille et Vilaine & 161 & $23,0 \%$ & $31 \%$ \\
\hline Morbihan & 122 & $17,4 \%$ & $22,72 \%$ \\
\hline Total & 701 & $100 \%$ & $100 \%$ \\
\hline
\end{tabular}

\footnotetext{
${ }^{5}$ Source: auteurs à partir des données LSA Expert et Insee en France métropolitaine, date arrêtée, octobre 2016
} 
L'échantillon de convenance, ne permet pas d'obtenir une répartition identique des bretons au sein des quatre départements.

\subsection{La mesure des concepts}

Rappelons que la fidélité est ici appréhendée dans sa dimension comportementale. Pour la mesurer, une question fut introduite dès le début du questionnaire et servira alors de question filtre lors des analyses ultérieures. Dans le contexte de grande consommation alimentaire, la fidélité étant intimement liée à la proximité d'accès (Bergadaà et Del Bucchia, 2009 ; Labbé Pinlon, Lombart et Louis, 2016), une question combinant fidélité et proximité d'accès fut introduite dès le début du questionnaire. En fin de questionnaire, le répondant est sondé sur ses intentions comportementales futures à travers un des quatre items de Zeithaml, Berry et Parasuraman (1996): la recommandation du drive. Le tableau 3 présente l'ensemble des questions relatives à la fidélité à l'enseigne.

\section{Tableau 3 : Mesure de la fidélité à l'enseigne}

\begin{tabular}{|l|l|}
\hline \multirow{3}{*}{ Vous fréquentez le drive : } & De votre magasin habituel \\
\cline { 2 - 2 } (plusieurs choix possibles) & D'un autre magasin proche de votre lieu de travail \\
\cline { 2 - 2 } & D'un autre magasin proche de votre domicile \\
\cline { 2 - 2 } & Autre : précisez \\
\hline Recommanderiez-vous ce & $\begin{array}{l}\text { Note de } 1 \text { à } 10: \text { «je ne le recommanderai pas du tout » à « je le } \\
\text { recommanderai très fortement » }\end{array}$ \\
\hline
\end{tabular}

La question relative à la fréquentation du drive admet plusieurs réponses possibles car lorsqu'un acheteur doit choisir son drive trois possibilités réelles se présentent à lui : fréquenter le drive de son magasin habituel et/ou privilégier un drive proche de son lieu de travail et/ou un drive proche de son domicile. Concernant le concept de proximité, les travaux fondateurs de Bergadaà et Del Bucchia (2009) démontrent le caractère multiple et complexe de ce dernier. Les auteurs identifient cinq proximités : la proximité d'accès, la proximité fonctionnelle, la proximité relationnelle, la proximité de processus ainsi que la proximité identitaire. Dans le cadre de cette recherche, seules les trois premières proximités furent étudiées, comme en rend compte le tableau 4.

Tableau 4 : Mesure de la proximité

\begin{tabular}{|c|c|c|}
\hline Concept mesuré & Question & Modalité de réponse \\
\hline \multirow[t]{2}{*}{ Proximité d'accès } & Satisfaction de la facilité d'accès & Note de 0 à 10 \\
\hline & $\begin{array}{l}\text { Estimation de la durée moyenne de votre } \\
\text { parcours pour accéder au drive }\end{array}$ & $\begin{array}{l}\text { Choix multiples : moins de } 10 \\
\text { minutes/ } 10 \text { à } 20 \text { minutes/ } 20 \text { à } 30 \\
\text { minutes/ plus de } 30 \text { minutes }\end{array}$ \\
\hline \multirow{8}{*}{$\begin{array}{l}\text { Proximité } \\
\text { fonctionnelle }\end{array}$} & Satisfaction du temps d'attente & Note de 0 à 10 \\
\hline & $\begin{array}{l}\text { Délai entre passation de la commande et sa } \\
\text { disponibilité }\end{array}$ & Note de 0 à 10 \\
\hline & Facilité d'authentification du drive & Note de 0 à 10 \\
\hline & Amplitude horaire & Note de 0 à 10 \\
\hline & Choix des créneaux de retrait proposés & Note de 0 à 10 \\
\hline & Choix des marques & Note de 0 à 10 \\
\hline & Assortiment de produits & Note de 0 à 10 \\
\hline & Accès aux promotions & Note de 0 à 10 \\
\hline
\end{tabular}




\begin{tabular}{|l|l|l|}
\hline & Prix pratiqués & Note de 0 à 10 \\
\hline & Suivi des dépenses & Note de 0 à 10 \\
\hline & Montant minimum de la commande & Note de 0 à 10 \\
\hline $\begin{array}{l}\text { Proximité } \\
\text { relationnelle }\end{array}$ & $\begin{array}{l}\text { Satisfaction envers la relation avec le } \\
\text { personnel du drive }\end{array}$ & Note de 0 à 10 \\
\hline
\end{tabular}

La proximité fonctionnelle, véritable axe de différenciation des drives par rapport aux formats traditionnels, a fait l'objet du plus grand nombre de questions. La proximité identitaire (mesure de l'adhésion aux valeurs de l'enseigne) semble plutôt faire l'objet d'une analyse à partir du point de vente lui-même plutôt que de son système de drive. Bien qu'élément important de la fidélité des consommateurs, la proximité de processus nécessite une bonne connaissance des pratiques du distributeur et se veut difficile à appréhender, particulièrement dans ce contexte.

\section{Résultats}

\subsection{La proximité du drive, un levier déterminant du comportement}

Sur l'ensemble des 701 questionnaires administrés, seuls 639 ont répondu à notre question filtre (Tableau 3). Ces 639 répondants constituent donc notre base de données de référence pour l'analyse des résultats. Parmi ces 639 répondants, 443 fréquentent le drive de leur magasin habituel.

Comme pour les points de vente physique (Bergadaà et Del Bucchia, 2009), la proximité d'accès demeure le facteur de choix primordial des drives. Il apparaît que le critère «proximité de mon domicile » est le critère dominant (sur l'ensemble des critères classés) : $50,9 \%$ des personnes interrogées le classent en première position. Cette recherche de proximité géographique est une préoccupation majeure des utilisateurs d'un drive car ceux-ci souhaitent maitriser leur temps en mettant en place une stratégie de rationalisation de leurs ressources temporelles (Mevel, Morvan, 2015). Or, la massification des achats de biens quotidiens, proposée par le drive, offre la possibilité de réduire le temps contraint. Les ménages souhaitent se déplacer de moins en moins fréquemment pour réaliser des achats qui apparaissent comme des activités sans grand intérêt. Il s'agit bien de créer une activité routinière et rationalisée (Picot-Coupey, Huré, Cliquet et Petr, 2009) et par conséquent de chercher à réduire les temps «morts » représentés par la fréquentation des grandes surfaces : temps de trajet, stationnement, ou encore déplacement dans le magasin (Gahinet, 2014). Implantés en sortie des grandes aires urbaines, ou encore à proximité des zones industrielles, sur l'axe travail-domicile, les drives représentent des commerces de transit.

La proximité d'accès telle que définie par Bergadaà et Del Bucchia (2009), demeure un critère de choix indéniable. Alors que traditionnellement le déplacement vers le point de vente est appréhendé comme un simple aller-retour depuis le domicile du consommateur, la mobilité croissante des individus impose de repenser les déplacements. Le trajet vers les points de vente doit être pensé comme faisant partie d'un chaînage de déplacements (Delage et Hani, 2014). Nous nous situons ici dans une logique d'optimisation des déplacements : le parcours vers le drive doit se penser comme faisant partie d'un tout et non plus comme se suffisant à lui-même. Le consommateur optimise ses déplacements : ainsi par exemple, va-t-il privilégier drive le plus proche de son parcours domicile-travail En effet, la proximité du lieu de travail apparaît en second critère de choix des consommateurs pour $26,7 \%$ des répondants, et la localisation sur l'axe domicile travail est classée en troisième position. La localisation des drives représente donc un avantage concurrentiel certain dans la fréquentation et le choix des unités de retrait par le consommateur. Les drives peuvent devenir un levier déterminant dans 
la stratégie d'optimisation du maillage territorial. Ils offrent aux réseaux une proximité géographique temporaire avec leur clientèle (Torre, 2009). Ces résultats montrent que l'implantation des drives doit respecter l'une des conditions de succès d'une implantation traditionnelle, à savoir le principe d'interception, ou comment accrocher le client?

L'analyse du taux de mixité montre que la majorité des consommateurs ne fréquente qu'un seul drive $(93,3 \%)$, et seuls $6,4 \%$ des sondés en fréquentent deux en complément de leur hypermarché ou supermarché habituel. Le drive comme format d'achat se montre donc plus captif que les formats traditionnels pour lesquels les clients multiplient les alternatives entre un magasin principal, un secondaire, puis des occasionnels.

\subsection{Proposition d'une typologie de «drivers » fidèles}

A l'issue de la question filtre, il apparaît que près de $70 \%$ des consommateurs fréquentent le drive de leur magasin habituel. Un tel résultat confirme que le drive consolide la relation de service établie par l'enseigne avec le client ; le client perçoit alors le drive comme un service complémentaire - une extension de son enseigne - ayant tendance à maintenir sa relation à l'enseigne qu'il fréquente habituellement. Cette fidélité des acheteurs de drive à leur enseigne semble conforter, de manière descriptive à ce stade, le transfert d'associations d'image entre l'enseigne et le drive.

Le tableau 5 rend compte des résultats quant à la probabilité de recommandation d'un drive de la part des usagers. Au regard des comportements prospectifs des consommateurs fidèles, 96,09\% recommanderaient leur drive (considérant que la recommandation débute à partir de 6/10 sur l'échelle), sachant que 53,29\% le recommanderaient très fortement. Ce résultat décrit l'importance en amont de la construction d'une relation positive avec l'enseigne et de sa capacité à transférer ces attributs au drive.

Tableau 5 : Résultats de la probabilité de recommander le drive

\begin{tabular}{|l|l|l|l|l|l|}
\hline & Echantillon & $\begin{array}{l}\text { Consommateurs } \\
\text { fidèles }\end{array}$ & & Echantillon & $\begin{array}{l}\text { Consommateurs } \\
\text { fidèles }\end{array}$ \\
\hline Note de 1 & $0 \%$ & $0 \%$ & Note de 6 & $5,26 \%$ & $3,18 \%$ \\
\hline Note de 2 & $0,31 \%$ & $0,44 \%$ & Note de 7 & $10,84 \%$ & $9,33 \%$ \\
\hline Note de 3 & $0,63 \%$ & $0,68 \%$ & Note de 8 & $30,14 \%$ & $30,29 \%$ \\
\hline Note de 4 & $0,31 \%$ & $0,22 \%$ & Note de 9 & $26,31 \%$ & $29,15 \%$ \\
\hline Note de 5 & $3,35 \%$ & $2,50 \%$ & Note de 10 & $22,80 \%$ & $24,14 \%$ \\
\hline
\end{tabular}

Afin d'apprécier plus finement la fidélité du client du drive, une classification ascendante hiérarchique est réalisée, de façon à regrouper des profils de consommateurs identiques dans leur évaluation du drive. L'analyse multidimensionnelle a été réalisée sur l'ensemble des 443 clients fidèles à leur enseigne habituelle parmi les 639 clients ayant répondu à cette question. Les profils ont été spécifiés selon le prisme des trois mesures de la proximité.

\subsubsection{Drive et proximité d'accès}

Le tableau 6 synthétise l'estimation de la facilité d'accès et montre que cette dernière affiche une note moyenne de 8,55. Cette bonne moyenne masque cependant d'importantes disparités au sein des clients. Les consommateurs du groupe 1 attribuent une note moyenne de 8,87 . Les utilisateurs du groupe 2 sont moins satisfaits que la moyenne avec une note de 7,67 sur ce critère. L'insatisfaction générale transpire chez les utilisateurs du groupe 3 sur l'ensemble des critères. Pour ces derniers, la facilité d'accès obtient une moyenne de 3,25. 
Tableau 6 : Estimation de la facilité d'accès (note sur 10)

\begin{tabular}{|l|c|}
\hline & Facilité d'accès routier \\
\hline Groupe 1 & 8,87 \\
\hline Groupe 2 & 7,67 \\
\hline Groupe 3 & 3,25 \\
\hline Moyenne & 8,55 \\
\hline
\end{tabular}

La proximité d'accès fut également appréhendée par une estimation de la durée moyenne du parcours retranscrite dans le tableau 7. En moyenne 57\% des utilisateurs estiment leur temps de trajet inférieur à dix minutes. Ce résultat rejoint la théorie (Bergadaà et Del Bucchia, 2009), selon laquelle la localisation joue un rôle important dans la fidélité.

Tableau 7 : Estimation de la durée moyenne du parcours (en pourcentage)

\begin{tabular}{|l|c|c|c|c|}
\hline & $\begin{array}{c}\text { Moins de 10 } \\
\text { minutes }\end{array}$ & $\mathbf{1 0}$ à 20 minutes & 20 à 30 minutes & Plus de 30 minutes \\
\hline Groupe 1 & 57,4 & 33,22 & 6,45 & 2,90 \\
\hline Groupe 2 & 51,31 & 34,21 & 11,84 & 2,63 \\
\hline Groupe 3 & 87,5 & 12,5 & - & -16 \\
\hline Moyenne & 57,78 & 31,55 & 8,50 & 2,16 \\
\hline
\end{tabular}

On notera toutefois quelques disparités selon les groupes d'individus. En effet, les individus du groupe 3 ne parcourent pas plus de vingt minutes et seuls 12,5\% ont un temps de trajet de 10 à 20 minutes de trajet. Pour le groupe 3 , la proximité d'accès explique par conséquent une grande partie de la décision de choix.

Les clients des groupes 2 et 3 consentent à parcourir un temps de trajet plus important. En effet, plus de $14 \%$ des individus du groupe 2 parcourent même plus de vingt minutes pour se rendre au drive. Si ces clients tirent un bénéfice évident de la proximité d'accès, leur satisfaction à l'égard du drive trouve également son origine dans d'autres éléments qui rend leur consentement à parcourir de la distance plus fort.

\subsubsection{Drive et proximité fonctionnelle}

Pour l'ensemble des critères relatifs à la proximité fonctionnelle (retranscrits dans le tableau 8), les clients en moyenne sont satisfaits de l'accessibilité et de la rapidité d'exécution du point de retrait du drive. Ces éléments confortent l'importance accordée par les clients au facteur temps.

Le tableau 8 souligne tout d'abord qu'en moyenne les clients fidèles sont satisfaits du processus de commande qui lui est proposé. Parmi les éléments les mieux notés nous retrouvons "le choix des créneaux proposés », créneaux pour lesquels l'information permet au client de connaitre les créneaux saturés tout en ayant à sa disposition un ensemble de créneaux nombreux et variés offrant la possibilité de récupérer ses achats à n'importe quel moment de la journée. «La confirmation de la commande par e-mail » est particulièrement appréciée des individus du groupe 1. Par contre, «l'accès aux promotions » est relativement moins bien noté. L'analyse des questions ouvertes montre que l'accès aux promotions est difficile et les promotions ne sont pas suffisamment mises en valeur par le site.

Tableau 8 : Principaux résultats de la proximité fonctionnelle (notes sur 10)

\begin{tabular}{|l|l|l|l|l|l|l|} 
Sélection & Accès & Montant & Choix des & Temps & Prix & La
\end{tabular}




\begin{tabular}{|l|l|l|l|l|l|l|l|}
\hline & $\begin{array}{l}\text { des } \\
\text { produits }\end{array}$ & $\begin{array}{l}\text { aux } \\
\text { promotions }\end{array}$ & $\begin{array}{l}\text { minimum } \\
\text { de la } \\
\text { commande }\end{array}$ & $\begin{array}{l}\text { créneaux } \\
\text { de retrait } \\
\text { proposés }\end{array}$ & d'attente & pratiqués & $\begin{array}{l}\text { confirmation } \\
\text { de commande } \\
\text { par e-mail }\end{array}$ \\
\hline Groupe 1 & 7,85 & 7,62 & 8,78 & 8,90 & 8,8 & 8,17 & 8,97 \\
\hline Groupe 2 & 6,55 & 6,14 & 6,82 & 7,18 & 7,36 & 6,01 & 8,05 \\
\hline Groupe 3 & 2,5 & 2,750 & 3,62 & 3,37 & 2,87 & 2,62 & 3,6 \\
\hline Moyenne & 7,40 & 7,06 & 8,19 & 8,47 & 8,37 & 7,56 & 8,59 \\
\hline
\end{tabular}

L'analyse des trois profils révèle que les clients du groupe 1 ont tendance à être plus satisfaits $(8,78)$ que la moyenne $(8,19)$ des éléments liés à la commande sur le site internet. Inversement, les individus du groupe 2 se situent juste en dessous de cette moyenne $(6,82)$. Enfin, le groupe 3 dans son ensemble représente des acheteurs plutôt insatisfaits des critères de proximité fonctionnelle proposés par le distributeur.

Alors qu'en moyenne les utilisateurs de drive accordent une note de 7,40 à la satisfaction de la sélection de produits proposés, le groupe 1 attribue une note supérieure de 7,85 à ce critère. Comme précédemment, les acheteurs du groupe 2 se montrent un peu moins satisfaits que la moyenne $(6,55)$ alors que le groupe 3 affiche à nouveau une satisfaction très faible sur l'ensemble services proposés par le drive $(2,5)$.

\subsubsection{Drive et proximité relationnelle}

Lorsque nous nous centrons sur le critère «relation avec le personnel du drive », moment privilégié de la relation, nous remarquons que celui-ci est très bien noté par les consommateurs sollicités. Ce résultat souligne le fait que les enseignes ont bien conscience que l'accueil du client par la personne chargée de la livraison au drive est une variable essentielle. Là encore d'importantes disparités apparaissent au sein de l'échantillon de clients fidèles. En effet, le groupe 1 attribue une note de 9,02 pour quantifier la relation positive avec le personnel du drive, le groupe 2 obtient une note moyenne de 7,94 tandis que ce résultat tombe à 2,87 pour les clients du groupe 3 .

Ce constat nous incite à étudier la fréquence d'achats en drive des clients interrogés. Le tableau 8 montre que les clients très satisfaits du groupe 1 utilisent plus fortement le drive que la moyenne : $6,45 \%$ d'entre eux y ont recours plusieurs fois par semaine et $56,12 \%$ une fois par semaine. A l'inverse, les acheteurs insatisfaits du groupe 3 semblent davantage occasionnels et la moitié d'entre eux n'utilise le drive qu'une fois par mois.

Tableau 9 : Fréquence d'achats en drive (en pourcentage)

\begin{tabular}{|l|l|l|l|l|l|l|}
\hline & $\begin{array}{l}\text { Puiseurs fois } \\
\text { par semaine }\end{array}$ & $\begin{array}{l}\text { Une fois par } \\
\text { semaine }\end{array}$ & $\begin{array}{l}\text { 2 à 3 fois par } \\
\text { mois }\end{array}$ & $\begin{array}{l}\text { Une fois par } \\
\text { mois }\end{array}$ & $\begin{array}{l}\text { Une fois tous } \\
\text { les 2 à 3 mois }\end{array}$ & $\begin{array}{l}\text { Moins } \\
\text { souvent }\end{array}$ \\
\hline Groupe 1 & 6,45 & 56,12 & 24,83 & 8,38 & 2,25 & 1,93 \\
\hline Groupe 2 & 2,63 & 48,68 & 18,42 & 17,10 & 7,89 & 5,26 \\
\hline Groupe 3 & - & 25 & 25 & 50 & - & - \\
\hline Moyenne & 3,30 & 53,23 & 24,60 & 11,94 & 3,88 & 3,02 \\
\hline
\end{tabular}

Ces résultats confortent l'analyse de Wallace, Giese et Johnson (2014) selon laquelle la stratégie multicanale des réseaux et la multiplication des services améliorent la satisfaction des consommateurs et par conséquent leur fidélité (comportementale et attitudinale).

\section{Discussion et implications managériales}

Cette recherche quantitative s'est intéressée aux acheteurs de drive alimentaire et à leur comportement de fidélité à leur enseigne. Nous nous sommes demandés si la relation en 
amont à l'enseigne se maintenait malgré le maillage intensif des enseignes à travers l'implantation de leurs drives. Autrement dit, les clients de drive restent-ils fidèles à leur enseigne habituelle malgré le large choix de drives proposés par les enseignes?

Ces comportements de fidélité ont été analysés par le prisme de la proximité d'accès, fonctionnelle et relationnelle. Les résultats confortent la théorie antérieure, appliquée au système de drive. La proximité d'accès combinée à la proximité fonctionnelle et relationnelle contribuent à la fidélité des acheteurs. Il apparaît que la majorité des acheteurs de drive se veulent fidèles à leur enseigne habituelle. Au regard de ces analyses, nous distinguons trois profils d'utilisateurs de drive fidèles à leur enseigne habituelle :

Le groupe 1 : les fidèles très satisfaits. Ces shoppers affichent une grande satisfaction, supérieure à la moyenne des utilisateurs de drive. Ce sont également des clients récurrents, très gros utilisateurs de ce service. Ils parcourent enfin des temps de trajet supérieurs aux deux autres groupes pour accéder à leur drive. Ils doivent donc faire l'objet d'une attention particulière de la part des distributeurs, d'autant plus qu'ils sont des prescripteurs auprès d'autres consommateurs, ce qui constitue une bonne arme anti-concurrence. Gagner et conserver des clients fidèles représente donc un enjeu majeur pour les entreprises de services en général et le commerce de détail en particulier. Peut-être les distributeurs peuvent-ils mettre en place une politique de récompense de fidélité particulière envers ces shoppers afin de les valoriser davantage et de manière différenciée ? Les distributeurs devront intensifier leurs efforts sur le marketing d'enseigne pour maintenir une image positive de leur drive (construite initialement par l'effet de halo avec l'enseigne) et bâtir une relation unique entre eux et ces clients pour qui la proximité ne semble pas être le facteur déterminant de leur venue en comparaison des autres groupes. Toutefois, il convient de mener une analyse plus en profondeur afin de mesurer le lien entre la fréquence et la très grande satisfaction. N'est-on pas en face d'un phénomène de rationalisation de la part de ces consommateurs qui cherchent de manière consciente ou non à rationaliser leurs nombreuses venues ? Enfin, ce résultat ouvre la voie à de nouveaux questionnements. Cela revient à lier le consentement à se déplacer des consommateurs à leur niveau d'implication et leur fidélité et donc à s'interroger sur le sens de la causalité. Les consommateurs affichent-ils un temps de trajet plus important parce qu'ils sont plus impliqués, ou bien la causalité est-elle inverse ? Quel rôle l'implication des consommateurs dans les achats au drive joue-t-elle dans le choix du point de retrait ? Quid alors de la proximité d'intégration du drive ? Ce concept mis en lumière par Schultz, Chaney et Debenetti (2016) suppose d'inscrire le drive dans son environnement local et replace la question du déploiement géographique des drives

Le groupe 2 : les fidèles juste satisfaits. Ces clients fidèles affichent néanmoins une satisfaction globalement plus faible que la moyenne des utilisateurs de drive. Après avoir identifié ces consommateurs, le distributeur doit préciser et comprendre leurs perceptions pour mener des actions correctives permettant d'élever leur niveau de satisfaction.

Le groupe 3 : les fidèles insatisfaits. Ces consommateurs de drives restent fidèles à leur enseigne habituelle, sans doute pour des raisons pratiques : plus de $87 \%$ d'entre eux estiment parcourir un temps de trajet inférieur à 10 minutes pour se rendre au drive. Ce résultat conforte l'idée selon lesquelles la localisation joue un rôle important dans la fidélité ainsi que la satisfaction et replace l'attraction gravitaire au centre des débats. En effet, bien qu'attirant une clientèle de flux, en mouvement, les zones de chalandise des drives ne s'appuieraientelles pas sur une attraction gravitaire, au même titre que les points de vente traditionnels? Dans cette perspective, l'inertie au changement (Meyer-Waarden, 2007), le coût d'un 
changement de magasin pour un consommateur peut expliquer que des clients non satisfaits soient fidèles à un magasin. En dépit de leur faible poids dans la clientèle globale des drives, ces clients doivent faire l'objet d'une attention particulière de la part des distributeurs. Ils sont sans doute très volatiles et sensibles à l'arrivée d'un nouveau concurrent à proximité.

Les résultats de cette recherche confirment par ailleurs que le drive peut être considéré comme un service complémentaire de l'enseigne, autrement dit, une extension de son enseigne puisque $70 \%$ des consommateurs interrogés fréquentent le drive de leur enseigne habituelle et plus de la moitié le recommanderait fortement. De là, le cadre théorique proposé en amont prenant appui sur les travaux de la psychologie cognitive se révèle justifié. Les contributions en effet des recherches dédiées à la catégorisation permettent, dans le cadre de cette recherche, de mieux appréhender le phénomène d'extension de l'enseigne en identifiant les transferts d'image entre l'enseigne et le drive, construisant ainsi un premier cadre théorique de l'image du drive. Cette approche perceptuelle est une première étape d'un modèle plus général du comportement du consommateur.

Toutefois, cette recherche ne questionne pas assez l'implication de l'acheteur de drive dans la nature et l'intensité de ses motivations. En effet, la motivation intrinsèquement liée au contexte d'achat influence le comportement du consommateur et certaines formes de recherche de produit (Rostchild, 1984), la recherche d'informations et donc le choix final (Muehling, Laczniak et Aandrews, 1993). Par ailleurs, la motivation d'achat revêt un caractère multidimensionnel, combinant la probabilité d'erreur, ou encore l'enjeu du risque perçu en cas de mauvais choix. De plus, la motivation se veut contingente de la nature même du produit (Assael, 1987, Valette-Florence, 1989). Certains achats alimentaires tels que le vin ou le chocolat revêtent un caractère hédonique fort, au même titre que l'achat de parfum alors que le signal envoyé ou le risque perçu diffèrent pour ces catégories de produits (Laurent et Kapfferer,1985). Dès lors comment évaluer au plus juste la motivation de fréquentation d'un drive combinant des produits identifiés comme des achats plaisirs tels que les yaourts ou encore le chocolat avec des achats au faible pouvoir hédonique tels que la lessive ou l'huile (Laurent et Kapfferer, 1985)? Il conviendrait de mettre en perspective les profils issus de cette recherche avec des profils de motivation. En l'occurrence ici, la motivation explique et influence le consentement des individus à se déplacer pour fréquenter un drive. Ainsi, une étude menée pour la Communauté Européenne appliquée à la distribution automobile (Lademann et Partner 2001) met en exergue le lien entre le consentement des individus à parcourir de la distance et l'implication de ces derniers Dès lors, il semble pertinent de s'interroger sur la valeur de magasinage apportée par le drive. A l'heure où les réseaux de distribution misent sur la valeur hédonique du magasinage et développent de véritables temples de la consommation, les drives semblent les parents pauvres de cette nouvelle organisation ? Au-delà de l'approvisionnement, cette nouvelle forme de distribution peut-elle répondre à d'autres motivations de fréquentation ? Le drive est-il voué à remplir une fonction utilitaire? A l'heure du shoptailnement, comment peut-il se combiner avec le ré-enchantement du magasinage? Quels sont les leviers d'action possibles que les réseaux peuvent actionner pour améliorer l'attachement envers une marque, en l'occurrence ici l'enseigne, préalable à toute comportement de fidélité (Lacoeuille, 2000) ? Quels bénéfices consommateurs les réseaux peuvent-ils mettre en place pour augmenter la proximité et la fidélité à l'enseigne à travers le drive? Une voie de recherche possible consiste à analyser le concept de magasinage et l'expérience clients au drive à travers le prisme de grille théorique développée par Fliser et Plichon (1984). 
A notre connaissance, aucune recherche ne s'est encore employée à délimiter et à formaliser le modèle perceptuel du drive représentant ainsi la première contribution de notre travail. Cette recherche nous encourage à poursuivre l'étude du drive en mesurant le modèle théorique proposé, prenant appui sur la construction de son image et déterminant causalement les comportements de fidélité des clients. Cette approche confirmatoire tiendra compte des résultats exploratoires et descriptifs de cette recherche en segmentant les clients selon leur niveau de satisfaction. La mesure de la satisfaction sera d'autant plus essentielle qu'ici les résultats montrent que même les clients insatisfaits sont fidèles. En cela, il devient pertinent d'approfondir la conceptualisation de la fidélité en incluant des variables attitudinales (rejoignant ainsi le travail de Jacoby et Kyner, 1973) de manière à renforcer l'explication de la fidélité à l'enseigne. Cette approche ouvrira les contributions des recherches passées montrant un lien direct et causal entre satisfaction et fidélité, cette fois, en traitant le modèle à l'inverse : l'insatisfaction et la fidélité.

\section{Bibliographie}

Alba J.W. et Hutchinson J.W. (1987), "Dimensions of consumer expertise”, Journal of Consumer Research, Vol. 13, p. 411-454.

ASSAEL H. (1987), "Consumer behaviour and marketing action, Boston Mass: Kent Publishing Co, p. 87.

BECK, N. et RYGL, D. (2015), " Categorization of multiple channel retailing in Multi-, Cross-, and Omni-channel for retailers and retailing", Journal of retailing and consumer services, Vol. 27, p. 170-178.

BERGADAA M. et DEL BUCCHIA C. (2009), «La recherche de proximité par le client dans le secteur de la grande consommation alimentaire », Revue management \& avenir, Vol. 21, p. 121-135.

BERRY L.L., (2000), "Cultivating service brand equity", Journal of the academy of marketing science, Vol. 28, No 1, p. 128-137.

BERRY, L.L., (1986), Retail Businesses are Service Businesses, Journal of Retailing, 62, 1, p. 3-6.

BEZES C. (2012), « La congruence perçue des magasins et du site internet : effets sur le choix du canal d'achat: le cas de la Fnac », Vie \& Sciences de l'entreprise, Vol. 190, p. 4670 .

BOUSH D. et LOKEN, B. (1991), "A process - tracing of brand extension evaluation", Journal of Marketing Research, Vol. 28, p. 16-28.

BURT S. et SPARKS L. (2002), "Corporate branding, internationalisation and the retailer as a brand", Corporate Reputation Review, Vol. 5, No 2, p. 194-212.

Cegarra J-J. et MerunKa, D. (1993), «Les extensions de marque : concepts et modèles » Recherche et Applications en Marketing, Vol. 8, No 1, p. 53-76.

CHANGEuR S. et CHANDON J-L., (1995), «Le territoire-produit : étude des frontières cognitives de la marque », Recherche et Applications en Marketing, Vol. 10, No 2, p. 31-51.

Colla E., Lapoule P., (2011), Les facteurs-clés du succès des cybermarchés : les enseignements du cas Tesco, Décision marketing, vol 61, p. 35-45.

Colla E. et LAPOUle P. (2012), «E-commerce: exploring the critical success factors », International journal of retail and distribution management, vol 40, ${ }^{\circ} 11, \mathrm{p} .842-$ 864. 
Colla E. et LAPOUle P. (2015), «Le drive: vecteur de cannibalisation ou de complémentarité ? Le cas de la grande distribution alimentaire », Revue Française du Marketing, Vol. 252, p. 55-70.

CHaudhuri A. et Holbrook M.B. (2001), "The chain of effects from brand trust and brand affect to brand performance: the role of brand loyalty", Journal of marketing, Vol. 65, No 2, p. 81-93.

Delage M. et Hani M. (2014), «Commerce et Mobilité », in Lemarchand N., Gasnier A. (dir), Le commerce dans tous ses états, PUR, Rennes.

DR. LADEMANN \&PARTNER (2001), Customer preferences for existing and potentiel sales and servicing alternatives in automobiles distribution, Hamburg.

DinNER I.M., VAN HEERDE H.J. et NeSLIN S.A (2014), "Driving online and offline sales: the cross channel effects of traditional, online display, and paid search advertising", Journal of marketing research, Vol 51, p. 527-545.

EHRENBERG A.S.C. (2000), Repeat-buying: fact theory and applications, nouvelle édition, Londres, Charles Griffin \& Company Ltd.

FILSER M. et PlichON V. (2004), «La valeur du comportement de magasinage. Statut théorique et apports au positionnement de l'enseigne », Revue française de gestion, Vol. 1, No 158 , p. 29-43.

FRY J.N., (1967), «Family branding and consumer choice », Journal of marketing Research, Vol. 4, p. 237-247.

GAHINET M. C. (2014), «Les nouveaux formats alimentaires de proximité : regards croisés des distributeurs et des consommateurs », Management et Avenir, Vol. 71., p.153-168.

GASNIER A. (2014), Corridors, centres commerciaux et retailtainement : une offre durable ?, Temps des courses, courses des temps, Lille, p. 187-198.

Heitz M., Douard J.P et Cliquet G. (2015), « Analyse des flux d'achat : l'aide à la localisation commerciale », Recherche et Applications en Marketing, Vol. 30, No 1., p. 118137.

JACOBY J. et KYNER D.B (1973), "Brand Loyalty Vs Repeat Purchasing Behavior", Journal of Marketing Research, Vol. 10, p.1-9.

LABBE-PINLON B., LOMBART C. et LOUIS D. (2016), « Impact de la proximité d'un magasin sur la fidélité des clients: le cas des magasins d'enseignes alimentaires de proximité », Revue Management \& Avenir, Vol. 84, p. 73-94.

LACOEUILHE J. (2000), L'attachement à la marque : proposition d'une échelle de mesure, Recherche et Application en Marketing, Vol. 15, No. 4, p. 61-77.

LAURENT G. ET KAPFERER J.N. (1985), Measuring consumer involvement profiles, Journal of marketing research, Vol. 22, p. 41-53.

MeVEl O. ET MoRVAN TH. (2015), «Drive, entropie et logistique urbaine : qu'attendre de la nouvelle relation de service initiée par les GMS avec les consommateurs ?», Logistique \& Management, Vol. 23, No2, pp. 21-30.

Mervis, C.B., Rosch, E., (1981), Categorization of natural objects, Annual Review of Psychology, $\mathrm{N}^{\circ} 32$, pp. 89-115.

MEYER-WAARDEN L. (2007), "The effects of loyalty programs on customer lifetime duration and share of wallet", Journal of Retailing, Vol. 83, No 2, p. 223-236.

MueHLing D.D., LACZNIAK R.N. ET ANDREWS J.C. (1993), Defining, operationalizing and using involvement in advertising research: a review, Journal of current issues and research in advertising, Vol. 15, No 1., p. 22-57.

PARK C.W., Milberg S. et LAWSON R. (1991), "Evaluation of brand extensions: the role of product feature similarity and brand concept consistency", Journal of Consumer Research, Vol. 18, p. 185-193. 
PAYNE A. et FROW P. (2004) "The role of multichannel integration customer relationship management", Industrial Marketing Management, Vol 33, p. 527-538.

Picot Coupey K., Huré et PIVETEAU L. (2016), " "Channel design to enrich customers' shopping experiences", International Journal of Retail and Distribution Management, Vol. 44, No 3, p. 336 - 368.

Picot Coupey K., Huré E., Cliquet G. et Petr C. (2009), "Grocery shopping and the Internet: exploring French consumers' perceptions of the hypermarket and cybermarket formats", The International Review of Retail, Distribution and Consumer Research, Vol. 19, No 4, p. 437-455.

PUnAKIVI M., YRJÖLÄ H., et HOLMSTRÖM J., (2001), "Solving the last mille issue: reception box and delivery box", International journal of physical distribution and logistics management, vol 31, ${ }^{\circ}$ 6, p. 427-439.

RANGaswamy A. et VAN BRUgGen G.H. (2005), "Opportunities and challenge in multichannel marketing", Journal of Interactive Marketing, Vol. 19, No 2, p. 5-11.

RosenBloOM B. (2007), "Multi-Channel strategy in Business to business market: prospects and problem", Industrial Marketing Management, Vol. 36, p 4-9.

RoTSCHILD C.R. (1984), Perspectives on involvement: current problems and future directions, NA-Advances in consumer research, No 11, p. 216-217.

ROUQUET A. et GOUDARZI K. (2010), «Le transfert d'activités logistique entrepriseconsommateur : une comparaison des cas Auchan Drive et IKEA, Logistique \& Management, Vol 18, No 2, p. 9-61.

Schultz M., Caney D. eT Debenedetti A. (2016), An integrative perspective of closeness in retailing: from retailers' sense-giving to consumers' sense-making, Journal of retailing and consumer services, Vol. 32, p. 218-226.

SECK A.M. (2010), «L'impact de l'émergence du multicanal sur la gestion de la distribution des services », Economies et sociétés, Vol. 44, No2, p. 231-250.

SeCK, A.M., Fulconis, F. et PACHE G. (2014), «Quels bénéfices peut retirer l'entreprise d'un management multicanal intégratif? », La Revue des sciences de gestion, Vol. 269-270, p. 55-64.

Sirdeshmukhet D., Singh J. et SABOl B. (2002), "Consumer, trust, value, and loyalty in relational exchanges", Journal of Marketing, Vol. 66, No 1, p. 15-37.

TORRE A., 2009, «Retour sur la notion de proximité géographique », Géographie, économie société, Vol. 11, p. 63-75.

TUCKER W.T. (1964), "The development of brand loyalty", Journal of Marketing Research, Vol. 1, No 3, p. 32-35.

TyBOUT A.M. et HAUSER J.R. (1981), “A marketing audit using a conceptual model of consumer behavior: Application and evaluation”, Journal of marketing, Vol. 45, pp. 82-101.

VALETTE-FloRENCE P. (1989), Conceptualisation et mesure de l'implication, Recherche et applications en marketing, Vol. 14, No. 1, p.57-78.

VAN BAAL S. et DACH C. (2005), "Free riding and customer retention across retailers' channels", Journal of interactive marketing, Vol. 19, No 2, p.75-85.

VANHEEMS R. (2009), "Distribution multicanal pourquoi les clients mixtes doivent faire 'objet d'une attention particulière?", Décisions marketing, Vol. 55, p. 41-52.

WALlace D.W., GIESE J.L. et JOHnson J.L. (2004), "Customer retailer loyalty in the context of multiple channel strategies", Journal of retailing, Vol. 80, p. 249-63.

ZEITHAML V.-A., BERRY L.-L. ET PARASURAMAN A. (1996), "The behavioral consequences of service quality", Journal of marketing, Vol. 60, No. 2, p. 31-46. 